\title{
3-D Convection Models: Are They Compatible with 1-D Models?
}

\author{
A. Nordlund \\ Theoretical Astrophysics Center and Astronomical Observatory, Juliane \\ Maries Vej 30, DK-2100 Copenhagen Ø, Denmark \\ R. F. Stein \\ Physics and Astronomy Department, Michigan State University, East \\ Lansing, MI 48824, USA
}

\begin{abstract}
We review properties of stellar convection, as derived from detailed 3-D numerical modeling, and assess to what extent 1-D models are able to provide a fair representation of stellar structure in various regions of the HR diagram. We point out a number of problems and discrepancies that are inevitable when using conventional 1-D models. The problems originate mainly in the surface layers, where horizontal fluctuations become particularly large, and where convective energy transport gives way to radiation. We conclude that it is necessary (and possible) to use three-dimensional models of these layers, in order to avoid the uncertainties and inaccuracies associated with 1-D representations.
\end{abstract}

\section{Introduction}

One-dimensional representations of stellar surface layers and envelopes have traditionally been the only option available for stellar modelers. In order to include the influence of convective energy transport in such models it is necessary to use simplified recipes, where the properties of convection are estimated from either local or non-local formulae, and where all quantities are one-dimensional; i.e., they are assumed to depend on radius alone. The classical example is Local Mixing Length theory (Böhm-Vitense 1958), but a number of more elaborate methods have also been put forward (see Canuto 1996 and references therein).

The one-dimensional treatments have a number of drawbacks. They are (explicitly or implicitly) parameterized, in many cases static and local, and radiative transfer of energy is either treated separately or only schematically. As a result, it is in practice necessary to calibrate the resulting models, using parameters either built into the description from the start, or parameters introduced specifically for the purpose of calibration. To the extent that it is at all possible to parameterize 3-D behavior within a $1-\mathrm{D}$ model representation, the relative simplicity of treatment and the associated low computational cost are the main advantages of 1-D representations.

Three-dimensional models are much more computer intensive, and do not allow the elaborate treatments of spectral line absorption that may be included in 


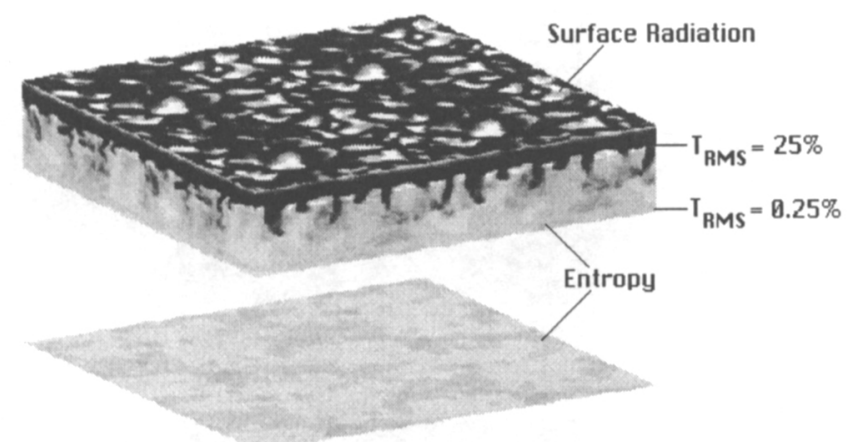

Figure 1. A perspective rendering of entropy fluctuations in the solar surface layers, as deduced from 3-D models (cf. Stein \& Nordlund 1998). The top surface shows the radiation intensity emergent from the top boundary of the model, while the drop-down copy of the bottom boundary illustrates the very weak entropy fluctuations there. Root mean square temperature fluctuations are also indicated.

1-D models, but instead have the advantage of being $a b$ initio, time-dependent, multi-dimensional, and non-local. With the steadily growing computational speed, the CPU-demands of these models are not prohibitive; in fact, lower resolution 3-D models that already in many respects are superior to $1-\mathrm{D}$ models have been around for about two decades (Nordlund 1980ab, 1982; Dravins, Lindegren, \& Nordlund 1981). Such models may today be computed in a few hours.

Here we briefly describe some key properties of convection in stellar surface layers, as deduced from 3-D models (Nordlund \& Stein 199a,b,c, Stein \& Nordlund 1998, Asplund et al. 1999a,b,c,d).

Additional figures that were shown in the talk are available through the internet: http://www.astro.ku.dk/“aake/talks/Budapest99.

\section{3-D Model Properties}

Figure 1 illustrates the structure of entropy fluctuations in the solar surface layers. As indicated in the figure, the entropy fluctuations correspond to root mean square temperature fluctuations of about $25 \%$ in the very surface layers (actual temperatures vary between about $4,000 \mathrm{~K}$ and $10,000 \mathrm{~K}$ ). Nevertheless, the temperature fluctuations near the bottom of the model (at a depth below the surface of some $2.5 \mathrm{Mm}$ ) are only $\sim 0.25 \%$, and are thus about two orders of magnitude smaller than at the surface.

The top face of the cube shows the emergent intensity; the bright cells and darker lanes between the cells constitute the "solar granulation": a cellular pattern of brightness fluctuations with a root mean square amplitude of some $20 \%$. Note that the intensity RMS is only about a fifth of what would be expected from an object with $25 \%$ temperature fluctuations emitting a black body radiation $\sim T^{4}$. It is the strong temperature sensitivity of the continuum opacity $\left(\kappa \sim T^{10}\right)$ that "hides" the strong temperature fluctuations. 


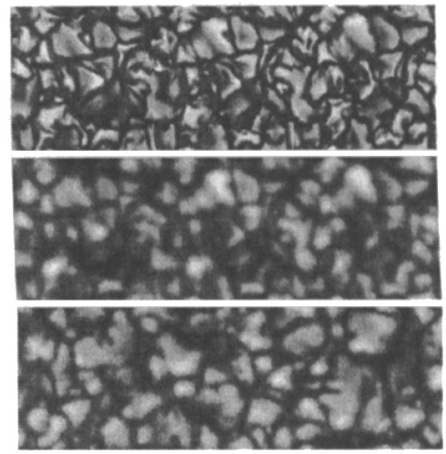

Figure 2. Synthetic and observed solar granulation. The top panel shows the surface radiation intensity computed from a $253 \times 253 \times 163$ simulation, while the middle panel shows the same pattern after folding with a point spread function that reduces the rms intensity fluctuation to about half, consistent with the rms intensity fluctuations of observations with the Swedish Solar Telescope at La Palma (lower panel-Lites, Nordlund, \& Scharmer 1989).

Fluctuations in other variables, such as density and velocity, also peak at the very surface. As discussed further below, these strong 3-D fluctuations give rise to significant alterations of the mean vertical stratification, effects that cannot be accounted for in 1-D models.

Figure 2 shows that numerical simulations with of the order of $200^{3}$ points produce surface intensity patterns in excellent agreement with high resolution observations. Quantitative statistical properties, such as power spectra and intensity histograms also agree with observations (Stein \& Nordlund 1998), although one may show that this is also true for some entirely artificial patterns (Nordlund et al. 1997). This illustrates the weakness of parameterized theories; agreement with observations does not prove that such theories are correct, or even useful. On the contrary, agreement between models and observations in one region of the HR diagram may be mistakenly taken as an indication that the same parameters will also produce accurate results in other regions of the HR diagram. Ludwig, Freytag \& Steffen (1999) have demonstrated that this is not the case, neither for the classical mixing length recipe, nor for the more elaborate theories by Canuto and coworkers.

One important reason for the difficulty of applying otherwise reasonably successful theories of convection to stars is the strong density stratification that characterizes the near-surface layers of stars. Below the surface the stratification is approximately polytropic, with a polytropic index much larger than that of an ideal gas, because of hydrogen and helium ionization. Above the surface the stratification is approximately exponential, because the temperature varies relatively little in the visible photosphere.

A characteristic and remarkable property of such strongly stratified convection is revealed by plotting entropy as a function of depth for a sample of horizontal points in a numerical model. As shown by Fig. 3, the upper envelope of the entropy distribution is almost totally flat. Indeed, ascending fluid is nearly 

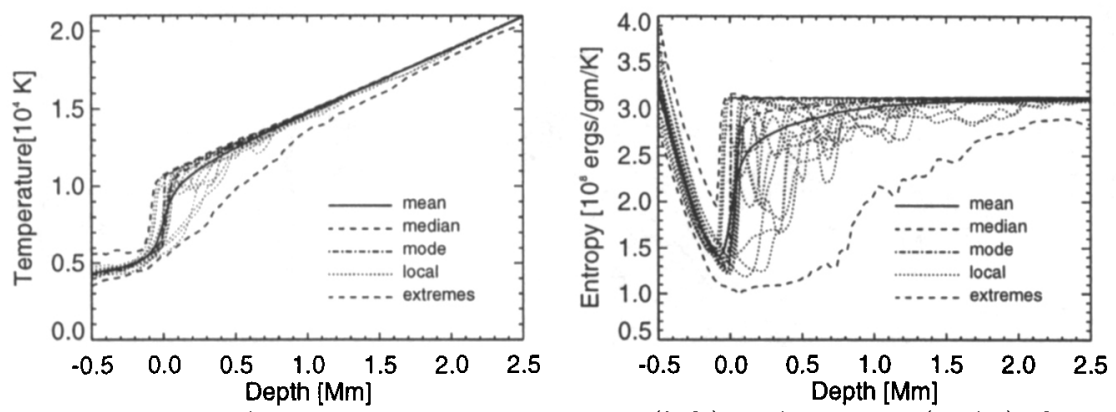

Figure 3. Fluctuations of temperature (left) and entropy (right), for a subsample of horizontal grid points in a numerical simulation (Stein \& Nordlund 1998)

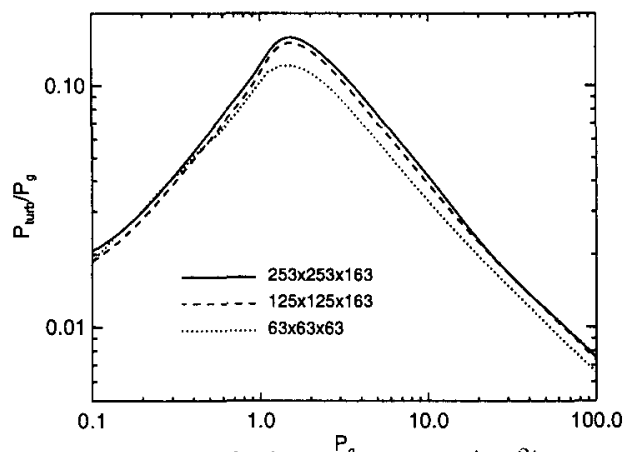

Figure 4. The ratio of turbulent pressure $\left\langle\rho u_{\mathrm{z}}^{2}\right\rangle$ to gas pressure $\left\langle P_{\mathbf{g}}\right\rangle$ as a function of depth for various numerical resolutions.

isentropic, while descending fluid has entropy fluctuations that decrease systematically with depth, asymptotically approaching the entropy of the ascending fluid. The reason for this is that mass conservation puts a severe constraint on the flow. Within one scale height, most of the ascending fluid must overturn, and since the surface layers of stars span a large number of scale heights, only a tiny fraction of the ascending fluid at some depth below the surface actually makes it to the top.

Conversely, the descending fluid is constantly "diluted" by overturning, nearly isentropic fluid. The contribution of low entropy fluid from the surface is thus rapidly overwhelmed by turbulent mixing with nearly isentropic fluid.

An effect, that in principle could be accounted for in 1-D models, is the levitation of the surface layers of stars by the turbulent pressure $\left\langle\rho u_{\mathrm{z}}^{2}\right\rangle$ (the average of the vertical component of the Reynolds's stress tensor). Because the convective velocities (as well as the corresponding Mach number) peak in the surface layers, the ratio of turbulent pressure to gas pressure also peaks there (see Fig. 4). Because of the rapid increase of density with depth, the turbulent pressure itself increases monotonically with depth. The gradient of the turbulent pressure therefore systematically contributes an upward directed force, that peaks in relative importance near the surface. The result is a "levitation" 


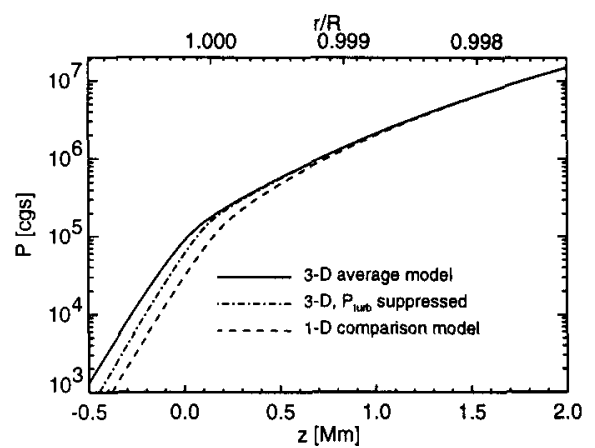

Figure 5. Pressure as a function of depth for an averaged 3-D model (solid line), for a comparison standard envelope model (dashed), and for a 3-D model where the gradient of the turbulent pressure has been artificially removed from the vertical pressure balance (dot-dashed).

of the photosphere that amounts to about half a scale height for the Sun (cf. Figs. 4-5, from Rosenthal et al. 1999).

Another effect, that does not have a correspondence in 1-D models, contributes to the levitation of stellar photospheres. The cause of this effect is the strong temperature sensitivity of the opacity in the surface layers already mentioned above. Gas that is hotter than average becomes very opaque, and is thus hidden from view, whereas cool gas is more transparent. The result is that the total surface radiation (the surface luminosity) is smaller for a 3-D model with a given relation $T(P)$ between horizontally averaged temperature and pressure than it would be for a 1-D model with the same $T(P)$ relation. A 3-D model with a specified surface luminosity (i.e., given effective temperature) must therefore have higher temperatures in the surface layers than a 1-D model with the same luminosity, and since higher temperatures correspond to larger vertical pressure scale heights, surfaces of constant pressure are displaced upwards in 3-D models.

As illustrated in Fig. 5, the total levitation is about a scale height in the case of the Sun. For giant stars, the effect is much larger (see Nordlund 1999 for the case of Procyon).

The ratio of turbulent pressure to gas pressure is roughly proportional to the root mean square Mach number. Even in the Sun, the distribution of Mach numbers extends beyond unity, i.e., in the horizontal layer where the ratio of turbulent to gas pressure peaks, supersonic velocities occur. This may appear surprising at first, but is to a large extent just a result of the surface cooling. Parcels of gas that move with subsonic velocities just below the surface become supersonic when the parcel passes through the optical surface, and temperature suddenly drops.

\section{Spectral Line Synthesis}

The fact that the relative turbulent pressure peaks in the very surface layers allows a direct test of the reliability of the 3-D models; the Doppler broadening of photospheric spectral lines is a direct manifestation of the velocity field in 


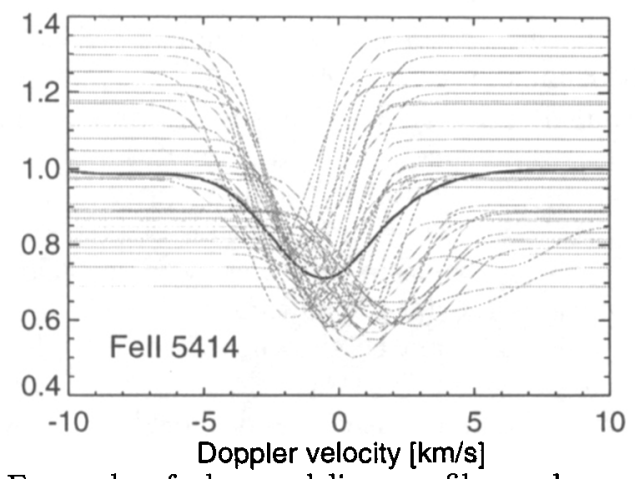

Figure 6. Example of observed line profile, and a subsample of the contributions from individual horizontal grid points in a snapshot.
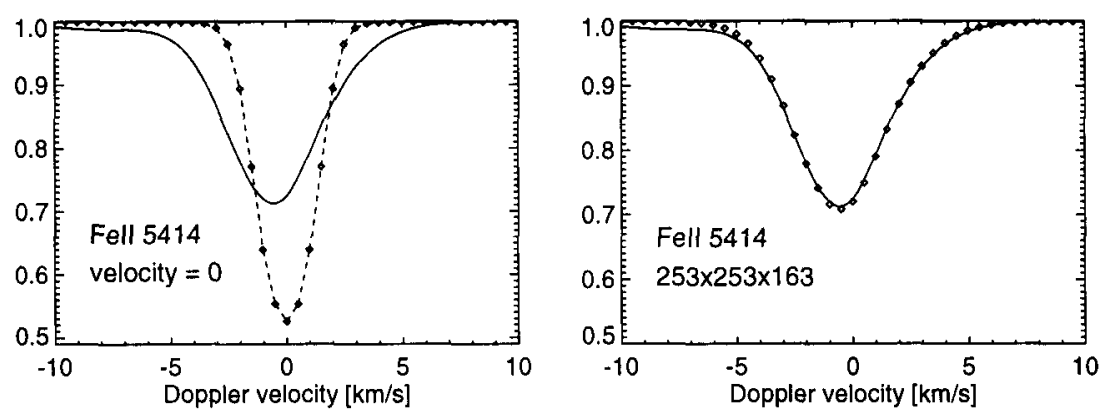

Figure 7. (left) The weak Fe II line $\lambda 5414$, as observed and as it would appear if there were no photospheric velocities. (right) Comparison of observed and synthetic Fe II $\lambda 5414$ spectral line profiles, based on the time average of synthetic spectral line profiles from a $253 \times 253 \times 163$ simulation.

these crucial layers. In particular, spectral lines from heavy atoms such as iron are significantly influenced by Doppler broadening. In traditional 1-D models this can only be accounted for by introducing arbitrary "micro-" and "macroturbulence" parameters. Micro-turbulence represents the small scale end of the turbulent spectrum and is applied to the spectral line absorption coefficient profile; it thus enhanced the total absorption (equivalent width) of the spectral lines. Macro-turbulence represents the large scale end of the turbulent spectrum, and is applied to the resulting spectrum; it increases the spectra line width (e.g., the full-width-at-half-maximum) without increasing the equivalent with.

Figure 6 illustrates the process by showing an example of spatially resolved spectral line profile from a 3 -D model of the solar surface layers. This should be compared to Fig. 7, where the left hand side panel shows the profile that would be obtained without any turbulent velocities, and the right hand side shows the match between synthetic and observed profiles that is obtained with a high resolution 3-D model of the solar surface layers. The spectral line is a weak Fe II line; these are formed somewhat deeper than the corresponding Fe I lines, and 
thus provide constraints on the velocity amplitudes as close as possible to the peak of the relative turbulent pressure (cf. Fig. 4).

Without the turbulent velocity field the spectral line profiles are all wrong; too narrow, too weak, with no spectral line asymmetry and no wavelength shift. With the turbulent velocity field obtained in sufficiently high-resolution models, the match to the spectral line shape and position is excellent (see Asplund et al. $1999 \mathrm{~b}, \mathrm{~d}$ ). Because of the correlation between vertical velocity and horizontal temperature fluctuations (surface brightness), there is a net ("convective") blue-shift of spectral lines. The match shown in Fig. 7 (right hand side panel) is obtained on an absolute wavelength scale; the absolute wavelength of solar spectral lines is known to high precision, and the absolute wavelength of the spectral line absorption profile is known to similar precision from laboratory measurements.

Spectral line shapes; widths, strengths, and asymmetries thus provide ideal "finger-prints" of convection in the surface layers of stars. As finger-prints they are very hard to forge; it would take a number of arbitrarily assigned parameters to obtain a good fit to just one of the observed spectral lines, and it is unlikely that the same set of parameters would results in a good fit for a different spectral line. In contrast, the 3 -D models contain no arbitrary parameters. In addition to the traditional parameters; effective temperature, surface acceleration of gravity, and chemical abundances, only the numerical resolution, and the associated treatment of viscous effects on scales below of a few grid zones enters in the numerical parameterization.

The excellent fit of the 3-D synthetic spectral lines links directly to relevant properties of the convection patterns; the spectral line width measures the vertical velocity amplitudes, the convective blue-shift measures the velocity-intensity correlation, and the spectral line asymmetry is the result of a complicated convolution of effects; correlations between velocity, temperature, and density, as reflected in the local spectral line absorption coefficients through the non-local effects of spectral line formation in a 3-D medium.

The fits of spectral line profiles down to fractions of a percent thus reflect the fact that models and reality obey the same set of fundamental dynamic equations, and show that, at least as far as the properties that control spectral line formation is concerned, the models have an adequate spatial resolution.

\section{Constraints from Helioseismology}

Complementary information about the properties of the crucial surface layers comes from the very accurate measurements of the frequencies of solar oscillations. The left hand side panel of Fig. 8 shows the scaled differences between the observed frequencies of solar oscillations and the ones obtained from classical 1-D envelope models. A systematic difference that depends mainly on frequency (thus indicating that the source of the difference is located in the surface layers) has been known for a long time. Rosenthal et al. (1999) showed that the differences in mean vertical structure between 3-D and 1-D models (as illustrated in Fig. 5) accounts for most of these discrepancies (cf. the right hand side panel of Fig. 8). Again, these effects are not parameterized, and are unavoidable consequences of the 3-D structure. The remaining differences may thus be 

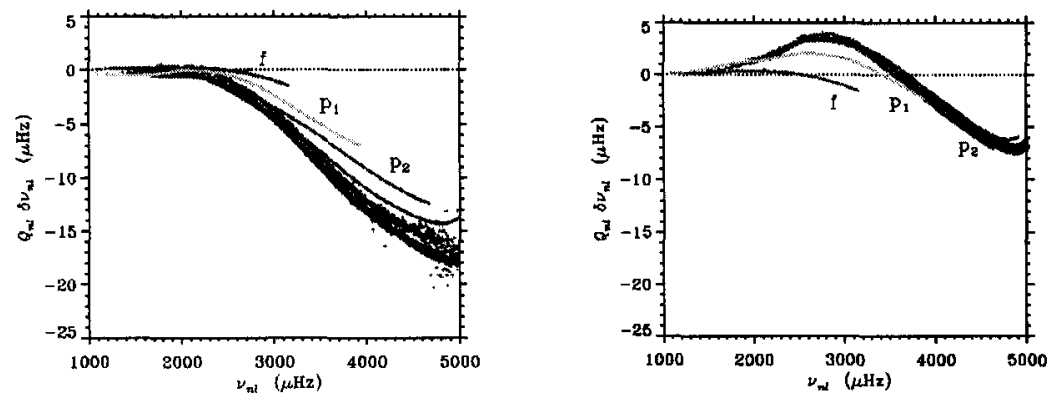

Figure 8. Scaled frequency differences between observations and a standard 1-D solar model (left), and between observations and a 1-D model constructed from horizontal averages of a 3-D model (right). From Rosenthal et al. (1999).

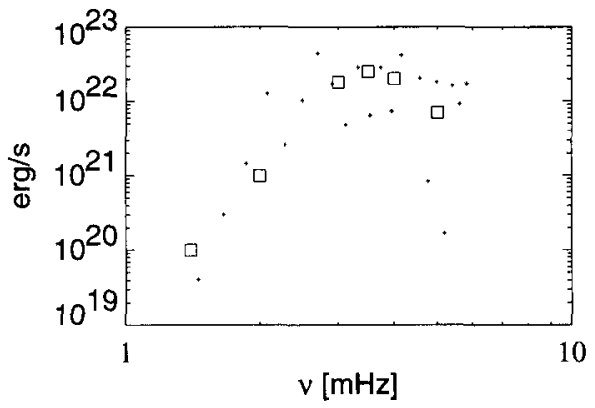

Figure 9. The stochastic energy input scaled to the total solar area. The small dots are from the numerical experiments, the large squares are determined from solar observations (Nordlund \& Stein 1999).

regarded as a diagnostic of the additional effects, such as 3-D wave propagation, non-adiabatic effects related to the strong horizontal variations of temperature, etc.

The 3-D models are also consistent with the excitation power and amplitude of the solar oscillations, as discussed by Nordlund \& Stein (1999). Figure 9 shows a comparison between the excitation power derived from 3-D models and the excitation power derived from the amplitudes and damping (as measured by the with of lines in the oscillation spectrum) of solar p modes.

The depth of the solar convection zone is known with high precision from helioseismological measurements $(0.287 R \pm 0.003 R$, Christensen-Dalsgaard, Gough, \& Thompson 1991; 0.287R $\pm 0.001 R$, Basu \& Antia 1997). As discussed by Rosenthal et al. (1999), the CZ-depth is to a large extent controlled by nearsurface properties. The equation of state is near-ideal in the deep layers of the convection zone, but is strongly influenced by ionization in the near-surface layers. Convection determines the "jump" in entropy at the surface. Photospheric spectral line blocking influences the relation between surface temperature and luminosity; about $12 \%$ of the continuum radiation is blocked by spectral lines 
in the solar spectrum; this raises the surface temperature by some $3 \%$, or about $200 \mathrm{~K}$, relative to a case with no spectral lines. When all of these factors are included, an excellent fit to the depth of the solar convection zone, $d=0.2885 R$, is obtained by patching a high-resolution 3-D model of the surface layers with a 1-D envelope models of the rest of the convection zone (Rosenthal et al. 1999).

\section{Discussion and Conclusions}

On the basis of comprehensive modeling of the Sun, where very accurate comparisons with observations may be made, one may conclude that 3-D numerical models of stellar surface layers provide quite accurate representations of the physics of these layers, and one may thus proceed to study and discuss the expected effects in other stars.

In cool main sequence stars, the convective overshoot into the visible photosphere is not properly accounted for in 1-D models. This is of particular importance in metal deficient stars, where the hydrodynamic cooling associated with overshoot cannot readily be balanced by spectral line heating, due to the sparsity of spectral lines. Asplund et al. (1999a) have shown that this may have a significant effect on the abundances derived from analysis of photospheric spectral lines. There are indications (including some that were presented at this meeting) that also the temperature structure of white dwarfs (which are even more sensitive to convective overshoot) may be significantly different from what is obtained with $1-\mathrm{D}$ models.

In hot (F-type) main sequence stars and giants, hydrogen ionization and recombination occurs in visible layers of the photosphere. As a consequence, temperature fluctuations (and opacity fluctuations even more so) become so large that it becomes highly questionable to compute the surface radiation using 1-D models. Certain discrepancies in observed color indices of F-stars are likely to be related to this (Gardiner, Kupka, \& Smalley 1999).

The low surface density of giant stars implies that the convective velocities must necessarily become increasingly supersonic in these stars (supersonic velocities occur even in the Sun's photosphere). Conventional 1-D recipes for estimating convective energy transport may be expected to become particularly inaccurate in this regime. Furthermore, in supergiants the size of convection cells starts becoming comparable to the entire stellar envelope, and it becomes necessary (and possible!) to model the star as a whole, rather than modeling only convection near the stellar surface.

In summary, one may conclude that 3-D modeling is crucial for a proper, qualitative and quantitative understanding of the surface layers of most stars. It is the only way to obtain robust, parameter-free estimates of the entropy jump that occurs near the surface of stars with a convective envelope, and it is the only reliable way to utilize the abundance of information that is encoded in the detailed shapes of photospheric spectral lines. For purposes of asteroseismology, 3-D modeling can yield reliable estimates of excitation power, and also of the photospheric elevation that must be accounted for to obtain accurate oscillation frequencies.

In conclusion, the answer to the question raised in the title of this contribution is that 3-D models are not compatible with 1-D models, and that an 
important task from the point of view of the main topic of this conference is to find practical ways to incorporate the results from the 3-D models into the modeling of pulsating stars. This might be achieved either by a (sufficiently clever and representative) parameterization of the 3 -D results (including the time dependence of horizontally averaged quantities), or else perhaps by "brute force" inclusion of 3-D models in the modeling of pulsating stars. It is worth emphasizing in this connection that the $N^{4}$ scaling of the computational expense of 3-D modeling that is normally considered a drawback, may be regarded as an advantage if one considers reduction of $N$. The robustness of thermal properties that one finds in the 3-D models may mean that it is better to use a low resolution, parameter-free 3-D model, than to attempt to parameterize overshoot, time dependence, non-locality, and so on.

Acknowledgments. The work of $\AA N$ was supported in part by the Danish Research Foundation, through its establishment of the Theoretical Astrophysics Center. RFS was supported by NASA grant NAG 5-4031 and NSF grant AST 9521785. Calculations were performed at the National Center for Supercomputer Applications, which is supported by the National Science Foundation, at Michigan State University and at UNI॰C, Denmark, which is supported by the Danish Natural Science Research Council.

\section{References}

Asplund, M., Nordlund, A., \& Trampedach, R. 1999a, A\&A, 346, L17

Asplund, M., Ludwig, H.-G., Nordlund, Å., \& Stein, R. F. 1999b, A\&A, (in preparation)

Asplund, M., Nordlund, A., \& Trampedach, R. 1999c, in ASP Conf. Ser. Vol. 173, Theory and Tests of Convection in Stellar Structure, ed. A. Giménez, E. F. Guinan, \& B. Montesinos (San Francisco: ASP), 221

Asplund, M., Nordlund, A., Trampedach, R., Allende Prieto, C., \& Stein, R. F. 1999d, A\&A (submitted)

Basu, S. \& Antia, H. M. 1997, MNRAS, 287, 189

Böhm-Vitense, E. 1958, ZfAp, 46, 108

Canuto, V. M. 1996, ApJ, 467, 385

Christensen-Dalsgaard, J., Gough, D. O., \& Thompson, M. J. 1991, ApJ, 378, 413

Dravins, D., Lindegren, L., \& Nordlund, Å. 1981, A\&A, 96, 345

Gardiner, R. B., Kupka, F., \& Smalley, B. 1999, A\&A, 347, 876

Lites, B. W., Nordlund, A., \& Scharmer, G. S. 1989, in NATO ASI C263, Solar and Stellar Granulation, ed. R. Rutten \& G. Severino (Dordrecht: Kluwer), 349

Ludwig, H. G., Freytag, B., \& Steffen, M. 1999, A\&A, 346, 111

Nordlund, $\AA .1980$ a, in Lecture Notes in Physics, Vol. 114, Stellar Turbulence, ed. D. F. Gray \& J. F. Linsky (Heidelberg: Springer), 17

Nordlund, A. 1980b, in Lecture Notes in Physics, Vol. 114, Stellar Turbulence, ed. D. F. Gray \& J. F. Linsky (Heidelberg: Springer), 213 
Nordlund, $\AA .1999$, http://www.astro.ku.dk/〜aake/talks/Budapest99

Nordlund, A., Spruit, H. C., Ludwig, H.-G., \& Trampedach, R. 1997, A\&A, 328, 229

Nordlund, A. \& Stein, R. F. 1997, in Solar Convection, Oscillations and their Relationship, ed. F. P. Pijpers, J. Christensen-Dalsgaard, \& C. S. Rosenthal (Dordrecht: Kluwer), 79

Nordlund, $\AA$. and Stein, R. F. 1999a, in ASP Conf. Ser. Vol. 173, Theory and Tests of Convection in Stellar Structure, ed. A. Giménez, E. F. Guinan, \& B. Montesinos (San Francisco: ASP), 91

Nordlund, A. \& Stein, R. F. 1999b, in Proc. IAU Symp. 185, ed. F. L. Deubner \& J. Christensen-Dalsgaard (Dordrecht: Kluwer), in press

Nordlund, A. \& Stein, R. F. 1999c, in ASSL Vol. 240, Proc. Internat. Conf. on Numerical Astrophysics 1998 (NAP98) ed. S. M. Miyama, K. Tomisaka, \& T. Hanagawa (Dordrecht: Kluwer), 293

Rosenthal, C. S., Christensen-Dalsgaard, J., Nordlund, A., Stein, R. F., \& Trampedach, R. 1999, A\&A, 351, 689

Stein, R. F. \& Nordlund, §. 1998, ApJ, 499, 914 